\title{
Z Research Square \\ Entropic evidence for a Pomeranchuk effect in Magic Angle graphene
}

Shahal Ilani ( $\square$ shahal.ilani@weizmann.ac.il)

Weizmann Institute of Science https://orcid.org/0000-0001-8589-7723

\section{Asaf Rozen}

Weizmann Institute of Science

Jeong Min Park

Massachusetts Institute of Technology

Uri Zondiner

Weizmann Institute of Science

\section{Yuan Cao}

Department of Physics, Massachusetts Institute of Technology https://orcid.org/0000-0002-90004501

\section{Daniel Rodan-Legrain}

Massachusetts Institute of Technology

\section{Takashi Taniguchi}

National Institute for Materials Science

\section{Kenji Watanabe}

National Institute for Materials Science https://orcid.org/0000-0003-3701-8119

\section{Yuval Oreg}

Weizmann Institute https://orcid.org/0000-0001-8753-8468

\section{Ady Stern}

Weizmann Institute of Science

\section{Erez Berg}

Weizmann Institute of Science

\section{Pablo Jarillo-Herrero}

Massachusetts Institute of Technology https://orcid.org/0000-0001-8217-8213

\section{Research Article}

Keywords: Pomeranchuk effect, Magic Angle graphene, analogous effect, twisted bilayer graphene

Posted Date: September 9th, 2020

DOl: https://doi.org/10.21203/rs.3.rs-72511/v1 
License: (c) (i) This work is licensed under a Creative Commons Attribution 4.0 International License. Read Full License

Version of Record: A version of this preprint was published at Nature on April 7th, 2021. See the published version at https://doi.org/10.1038/s41586-021-03319-3. 


\section{Entropic evidence for a Pomeranchuk effect in magic angle graphene}

Asaf Rozen ${ }^{1+}$, Jeong Min Park ${ }^{2+}$, Uri Zondiner ${ }^{1^{\dagger}}$, Yuan $\mathrm{CaO}^{2+}$, Daniel Rodan-Legrain ${ }^{2}$, Takashi Taniguchi ${ }^{3}$, Kenji Watanabe ${ }^{3}$, Yuval Oreg ${ }^{1}$, Ady Stern ${ }^{1}$, Erez Berg ${ }^{1 *}$, Pablo JarilloHerrero ${ }^{2 *}$ and Shahal Ilani ${ }^{1^{*}}$

${ }^{1}$ Department of Condensed Matter Physics, Weizmann Institute of Science, Rehovot 76100, Israel.

2 Department of Physics, Massachusetts Institute of Technology, Cambridge, Massachusetts 02139, USA.

${ }^{3}$ National Institute for Materials Science, 1-1 Namiki, Tsukuba, 305-0044 Japan.

${ }^{\dagger}$ These authors contributed equally to the work.

*Correspondence to: erez.berg@weizmann.ac.il,pjarillo@mit.edu, shahal.ilani@weizmann.ac.il

In the 1950 's, Pomeranchuk ${ }^{1}$ predicted that, counterintuitively, liquid ${ }^{3} \mathrm{He}$ may solidify upon heating, due to a high excess spin entropy in the solid phase. Here, using both local and global electronic entropy and compressibility measurements, we show that an analogous effect occurs in magic angle twisted bilayer graphene. Near a filling of one electron per moiré unit cell, we observe a dramatic increase in the electronic entropy to about $1 \boldsymbol{k}_{B}$ per unit cell. This large excess entropy is quenched by an in-plane magnetic field, pointing to its magnetic origin. A sharp drop in the compressibility as a function of the electron density, associated with a reset of the Fermi level back to the vicinity of the Dirac point, marks a clear boundary between two phases. We map this jump as a function of electron density, temperature, and magnetic field. This reveals a phase diagram that is consistent with a Pomeranchuk-like temperature- and fielddriven transition from a low-entropy electronic liquid to a high-entropy correlated state with nearly-free magnetic moments. The correlated state features an unusual combination of seemingly contradictory properties, some associated with itinerant electrons, such as the absence of a thermodynamic gap, metallicity, and a Dirac-like compressibility, and others associated with localized moments, such as a large entropy and its disappearance with magnetic field. Moreover, the energy scales characterizing these two sets of properties are very different: whereas the compressibility jump onsets at $T \sim 30 \mathrm{~K}$, the bandwidth of magnetic excitations is $\sim 3 \mathrm{~K}$ or smaller. The hybrid nature of the new correlated state and the large separation of energy scales have key implications for the physics of correlated states in twisted bilayer graphene. 
Systems of strongly interacting fermions exhibit a competition between localization, minimizing the potential energy, and itineracy, minimizing the kinetic energy. The advent of two-dimensional moiré systems, such as magic angle twisted bilayer graphene $\mathrm{e}^{2-6}$ (MATBG), opens a new route to study this physics by controlling the ratio between the electronic interactions and bandwidth in a highly tunable artificial lattice. In systems where this ratio is large, such as transition metal dichalcogenides hetero-bilayers, electrons tend to localize to the lattice sites, forming Mott insulators ${ }^{7,8}$. In the other extreme, where the electronic bandwidth is large, as in bilayer graphene with a large twist angle, a Fermi liquid state is formed in which electrons are itinerant. MATBG provides a fascinating example of a system at the boundary between these two extremes. This system shows a host of electronic phases, including correlated insulators ${ }^{3,9,10}$, Chern insulators $^{11-13}$, superconductors ${ }^{4,9,10}$, and ferromagnets ${ }^{14,15}$. Scanning tunneling spectroscopy ${ }^{16-19}$ and electronic compressibility measurements ${ }^{20,21}$ indicate that in this system Coulomb interactions and kinetic energies are indeed comparable. In this regime, there is an inherent tension between localized and itinerant descriptions of the physics. Moreover, the growing understanding that the nearly-flat bands in MATBG have a topological character $22-24$ implies that a simple "atomic" description, in which electrons are localized to individual moiré lattice sites, may not be appropriate. Instead, a picture analogous to that of quantum Hall ferromagnetism has been proposed as an alternative starting point ${ }^{25-27}$. Understanding this interplay between itineracy and localization, and the new physics that emerges from it, remains a major challenge.

In this work we find that, surprisingly, the correlated state in MATBG above a filling of one electron per moiré site has a hybrid nature, with some of its properties resembling those of an itinerant system, and others which are usually associated with localized electrons. Measurements of the electronic entropy at temperatures as low as a few Kelvin reveal that this state has an unusually large excess entropy, which is rapidly suppressed by a moderate in-plane magnetic field. This suggests that even at such low temperatures, there are strongly fluctuating magnetic moments in the system, a behavior that is typically 
associated with local moments. On the other hand, our measurements find that this state is metallic and has no thermodynamic gap nearby, which is naturally understood within an itinerant picture.

The presence of fluctuating moments at temperatures that are much smaller than the electronic bandwidth indicates the existence of a new, anomalously small energy scale associated with the bandwidth of magnetic excitations, which is an order of magnitude smaller than the energy scale where a jump appears in the compressibility ${ }^{21,28}$. This jump marks the boundary between the new state at $v>1$ and the more familiar state at lower densities. By tracking the dependence of this boundary on temperature and magnetic field, we find that it exhibits an electronic analogue $29-31$ of the famous Pomeranchuk effect ${ }^{1}$ in ${ }^{3} \mathrm{He}$. In that system, a transition from a Fermi liquid to a solid occurs upon increasing temperature, driven by the high spin entropy of the localized atoms in the solid. Similarly, we find that the new state above $v=1$ is favored relative to the metallic state at $v<1$ upon raising the temperature, due the former's high magnetic entropy. The same transition can also be driven by an in-plane magnetic field, due to the energy gain associated with polarizing the free moments. The existence of this hybrid state, with its itinerant electrons coexisting with strongly fluctuating magnetic moments, calls for a new understanding of electron correlations in MATBG.

The data reported here is measured using two independent techniques on two conceptually different devices. The bulk of the results are obtained from local measurements of the electronic entropy ${ }^{32,33}$ and compressibility using a scanning nanotube single-electron transistor (SET) on hBN-encapsulated twisted bilayer device (Device 1, Fig. 1a). We focus on a spatial region whose twist angle is close to the theoretical magic angle, and is homogenous over a large area $(5 \mu \mathrm{m} \times 4 \mu \mathrm{m})$ to within the third digit, $\theta=1.130 \pm 0.005$. Similar results are obtained from global entropy measurements using a monolayer graphene sensor to detect the chemical potential of MATBG (Device 2, Fig. 3a). Both methods have been described elsewhere ${ }^{21,34}$. 
The inverse compressibility, $d \mu / d n$, measured in Device 1 at $T=15 \mathrm{~K}$ as a function of the filling factor, $v=n /\left(n_{s} / 4\right.$ ) (where $n_{s}$ corresponds to four electrons per moiré unit cell), is shown in Fig. 1b. As reported previously ${ }^{21}$, sharp jumps in $d \mu / d n$ are observed close to integer $v^{\prime} s$, where the system rapidly evolves from high to low compressibility, reflecting an abrupt reconstruction of the Fermi surface. These were termed Dirac revivals since they were interpreted as resets of partially filled energy bands back to near charge neutrality, leading to the decreased compressibility. As seen in the figure, the cascade of revivals is already very prominent at these relatively high temperatures (i.e. above typical correlated insulator and superconducting critical temperature scales). Measurements of $\rho_{x x}$ vs. $v$ at various temperatures (Fig. 1c) show insulating behavior at $v=2$ and semimetallic behavior at $v=0$. As previously-noted ${ }^{35}, \rho_{x x}$ shows a step-like increase across $v \approx 1$ at high temperatures, and this feature gradually disappears with lowering the temperature (although a small peak in the resistivity close to $v=1$ remains). This behavior is different from the insulating behavior observed at other integer filling factors.

The unusual physics of the electronic state around $v=1$ is revealed by measuring the dependence of the inverse compressibility, $d \mu / d n$, on temperature, $T$, and parallel magnetic field, $B_{\|}$. In Fig. 2a we examine the temperature dependence of $d \mu / d n$ near $v=1$ at $B_{\|}=0 \mathrm{~T}$. At low temperature, a jump ${ }^{21}$ in $d \mu / d n$ occurs at a filling factor slightly larger than 1 . Increasing the temperature moves the jump towards a lower filling factor, and surprisingly, increases the magnitude of the jump, rather than smearing it. A similar measurement with $B_{\|}=12 \mathrm{~T}$ is shown in Fig. $2 \mathrm{~b}$. Compared to $B_{\|}=0 \mathrm{~T}$, at low $T$ the jump is much larger and closer to $v=1$. Increasing the temperature at $B_{\|}=12 \mathrm{~T}$ maintains the jump close to $v=1$ and, oppositely to the $B_{\|}=0 \mathrm{~T}$ case, reduces its amplitude and increases its width.

The chemical potential, $\mu(\nu, T)$ (measured relative to that at the charge neutrality point), can be obtained by integrating $d \mu / d n$ over the density at different temperatures (Fig. 2c,d). We see that $\mu$ has a strong temperature dependence for a certain range of $\nu^{\prime}$ s. This is clearly seen when we plot $\mu$ vs. $T$ at two representative $v^{\prime}$ s (Fig. 2c, inset). At $v=$ 
$0.2, \mu$ is practically independent of $T$ (blue). In contrast, at $v=0.9$ (red) we see that $\mu$ is nearly constant until $T \sim 4 \mathrm{~K}$, after which it starts to decrease approximately linearly with T. At $v>1.15, \mu$ is again nearly temperature independent. Comparing $\mu$ at $B_{\|}=0 \mathrm{~T}$ (Fig. 2c) and $B_{\|}=12 \mathrm{~T}$ (Fig. $2 \mathrm{~d}$ ) reveals a clear contrast: whereas for $B_{\|}=0 \mathrm{~T}$, $\mu$ is a decreasing function of temperature for $0.4<v<1.15$, for $B_{\|}=12 \mathrm{~T}$, $\mu$ decreases with $T$ for $v<$ 0.9 and increases for $v>0.9$.

Measurements of the temperature dependence of $\mu$ allow us to directly determine the entropy of the system, by integrating Maxwell's relation: $\left(\frac{\partial s}{\partial v}\right)_{T}=-\left(\frac{\partial \mu}{\partial T}\right)_{v}$, to obtain $s(v, T)$ (where $s$ is the entropy per moiré unit cell). For more details on the procedure of extracting the entropy, see Supplementary Information section SI1. Fig. 2e shows $s(v)$ at $T \approx 10 \mathrm{~K}$ (obtained by extracting the slope of $\mu$ vs. $T$ in the range $T=4.5 \mathrm{~K}-15 \mathrm{~K}$ ), for $B_{\|}=0 \mathrm{~T}, 4 \mathrm{~T}, 8 \mathrm{~T}$, and $12 \mathrm{~T}$. At $B_{\|}=0 \mathrm{~T}$ the entropy is small at low $v^{\prime}$ s, climbs close to $v=1$, and remains roughly constant between $v=1$ and 2 at about $1.2 k_{B}$ per unit cell. Near $v=2$ the entropy has a sharp drop, and it starts decreasing towards zero after $v=$ 3. We note that the dependence of the entropy on $v$ is qualitatively different from that of the compressibility. Specifically, whereas the compressibility drops sharply near $v=1$ (Fig. 2a), the entropy does not drop, but rather remains at a high value.

An important insight into the origin of this large entropy can be gained by examining its magnetic field dependence. As seen in Fig. 2e, the entropy above $v=1$ depends strongly on $B_{\|}$. In particular, at $B_{\|}=12 \mathrm{~T}$, most of the entropy between $v=1$ and 2 is quenched. The inset shows $s\left(B_{\|}=0 \mathrm{~T}\right)-s\left(B_{\|}=12 \mathrm{~T}\right)$ vs. $v$ (with errorbars indicated by the purple shading; see Supplementary Information SI1). The entropy difference increases sharply near $v=1$, reaching a maximum of $0.9 \pm 0.1 k_{B}$ between $v=1$ and 2 . To appreciate the significance of this value, recall that an entropy of $k_{B} \ln (2) \approx 0.7 k_{B}$ corresponds to two degenerate states on each moiré unit cell. Moreover, in a Fermi liquid, we would expect a much weaker change of the entropy with $B_{\|}$(Supplementary Information SI4), of the order of $k_{B}$ times the ratio of the Zeeman energy (about $1 \mathrm{meV}$ at $B_{\|}=12 \mathrm{~T}$ ) to the bandwidth, estimated to be $W \sim 30 \mathrm{meV}$ (see below). Finally, we 
observe that the entropy vs. $v$ at $B_{\|}=12 \mathrm{~T}$ shows a cascade of drops following each integer $v$, similar to the cascade of revivals seen in the compressibility (Supplementary Info. SI5), as obtained in a mean-field calculation (Supplementary Info. SI3). The dramatic quenching of entropy by a moderate in-plane magnetic field is strongly suggestive of its magnetic origin.

To test the robustness of our results, we measured the entropy in a completely different setup, in which a sheet of monolayer graphene is used to sense the chemical potential of the MATBG, averaged over the entire device ${ }^{34}$ (Fig. 3a). Fig. 3b shows the entropy extracted in three different temperature ranges. In particular, we see that the entropy from global measurements done in the range $T=4 \mathrm{~K}-16 \mathrm{~K}$ (blue curve, Fig. $3 \mathrm{~b}$ ) is in good agreement with the locally measured one over a similar range of temperatures (see inset for a comparison). The agreement is both in the overall shape and magnitude of $s(v)$, and in detailed features, such as the step-like drop near $v=2$. The global measurement setup further extends the experiment to the high temperature regime. As seen in Fig. 3b, upon raising the temperature, the minimum in the entropy at $v=0$ gradually fills in, evolving from a double-dome structure at low $T$ (corresponding to the valence and conduction flat bands) to a single dome at high $T$. This high temperature dependence can be qualitatively reproduced by a naïve calculation for a system of noninteracting electrons in Dirac bands, whose density of states rises linearly from the charge neutrality point until the band edges (Fig. 3c). The merging of the domes in $s(v)$ occurs when the temperature exceeds some fraction of the bandwidth. Calibrating the bandwidth in the calculation using the measured entropy at $T \approx 55 \mathrm{~K}$ gives $W \approx 30 \mathrm{meV}$ (where $W$ is the full bandwidth - from valence band bottom to conduction band top), in rough agreement with the values deduced from STM ${ }^{16-19}$ and compressibility ${ }^{34}$ experiments. Of course, we do not expect the free-electron picture to apply at low temperatures, since there interactions change the physics dramatically. We note that the measured $s(v)$ in the valence band (Fig. 3b) is approximately a mirror image of $s(v)$ in the conduction band, although it is somewhat smaller and has less pronounced features. 
This is consistent with the fact that the observed cascade of revivals in the inverse compressibility is weaker in the valence band relative to that in the conduction band ${ }^{21,34}$.

Our results so far show that a dramatic change occurs in the compressibility and the entropy near $v=1$. The compressibility experiences an abrupt drop at the revival transition (Fig. 2a,b), and at approximately the same filling, the magnetic-field-dependent part of the entropy sharply rises (Fig. 2e, inset). This rapid change may be due to a continuous buildup of electronic correlations. Alternatively, it can be interpreted as an underlying first-order phase transition between two distinct phases. Naively, one would then expect a discontinuous jump in thermodynamic properties and hysteretic behavior across the transition, which are not observed. However, we note that a true first-order phase transition can never occur in two dimensions in the presence of disorder or longrange Coulomb interactions ${ }^{36}$, as these will always broaden the transition into a mesoscale coexistence region. Experimentally, since the observed compressibility revival feature is relatively sharp, it can be precisely tracked, mapping a phase diagram as the function of temperature and magnetic field. Below, we show that interpreting this revival feature as a proxy for a first-order transition naturally explains much of the underlying physics.

We define the filling factor $v_{R}$ of the revival feature as the midpoint of the sharp rise in $d \mu / d n$ (tracking the beginning or the end of the rise leads to similar conclusions, see Supplementary Info. SI5). As we have seen in Fig. 2a, raising the temperature leads to an observable change in $v_{R}$. A similar measurement of $d \mu / d n$ vs. $v$ at different magnetic fields (Fig. 4a) shows that $v_{R}$ also shifts with $B_{\|}$. The measured locations of the revival feature as a function of $B_{\|}$and $T$ form a surface in the $\left(\nu, B_{\|}, T\right)$ space, shown in Fig. $4 \mathrm{~b}$. Projections of this surface onto the $\left(v, B_{\|}\right)$and $(v, T)$ planes are presented in Figs. $4 c, d$. Examining the magnetic field dependence of $v_{R}$ (Fig. 4c), we see that at $T=2.8 \mathrm{~K}, v_{R}$ is weakly dependent on $B_{\|}$at low fields, but starts to decrease linearly with field above $B_{\|} \approx$ 4T. At higher temperatures, $v_{R}$ is similarly insensitive to magnetic field at low $B_{\|}$, and 
decreases with increasing $B_{\|}$at higher fields. The crossover field between the two behaviors increases as the temperature increases.

Another interesting aspect of the evolution of $v_{R}$ is highlighted by looking at its temperature dependence at the different magnetic fields (Fig. 4d). At $B_{\|}=0$ T (blue) $v_{R}$ is linear in $T$ at low temperatures, and curves up at higher temperatures. As the magnetic field increases, the entire curve shifts towards smaller $v^{\prime}$ s, and simultaneously its slope at low temperatures changes sign. At the highest field, $B_{\|}=12 \mathrm{~T}, v_{R}$ first increases with temperature, reaches a maximum at $T \approx 9 \mathrm{~K}$, and then decreases at a higher $T$.

The phenomenology seen in Figs. $4 b-d$ can be understood within a simple interpretation, in terms of a first-order phase transition at $v=v_{R}$ between two phases: a Fermi liquid phase below $v_{R}$, and a 'free moment' phase above it. The latter phase has a high concentration of free moments (of the order of one per moiré site), coexisting with a low density of itinerant electrons. Within this framework, the movement of the transition point $v_{R}$ as a function of $B_{\|}$and $T$ reflects the magnetization and entropy differences between the two neighboring phases.

At $B_{\|}=0 \mathrm{~T}$, the free moment phase has a higher entropy than the Fermi liquid, due to thermal fluctuations of the moments. Hence, the former phase becomes increasingly entropically-favorable the higher the temperature. This explains the observed decrease of $v_{R}$ with increasing $T$ at low fields (Fig. $4 \mathrm{~d}$ ). Raising the temperature at a fixed $v$ may therefore drive a transition from the Fermi liquid to the free moments phase, an electronic analogue of the Pomeranchuk effect. As $B_{\|}$increases and the Zeeman energy exceeds the temperature, the moments become nearly fully polarized and their entropy is quenched (as is observed directly in Fig. 2e). Consequently, one expects that at low temperatures and sufficiently high fields, the Fermi liquid phase would be favored by raising the temperature, but that this trend will reverse once the temperature becomes larger than the Zeeman energy. This explains the non-monotonic behavior of $v_{R}$ as a function of $T$, seen at $B_{\|}=12 \mathrm{~T}$ in Fig. $4 \mathrm{~d}$. The main features of the phase boundary are qualitatively reproduced in an explicit model for the thermodynamics of the two phases 
(see Supplementary Info. SI7), as shown in the insets of Figs. 4b,c,d. We note that, although this simple model of a transition between a Fermi liquid and a localized moment phase explains much of the phenomenology present in Fig. 4, a complete understanding requires a more detailed consideration of interactions also for $v<v_{R}$ (e.g. to account for negative compressibility, revival strength, etc) $)^{21,34}$.

To conclude, our measurements reveal the emergence of a large entropy above $v=$ 1. This excess entropy is suppressed upon the application of a magnetic field, highlighting its magnetic origin. We observe two regions with vastly different properties on either side of $v=1$. We map the boundary between these two regions, and show that it can be naturally understood as a transition from a Fermi liquid-like phase to a free moment phase. The latter has several highly unusual characteristics: its moments remain strongly fluctuating down to very low temperatures (3K or less), much lower than the electronic bandwidth and the onset temperature of the revival feature in the compressibility. Moreover, this phase is compressible and metallic, with no sign of a thermodynamic gap. These observations raise important challenges for our understanding of electron correlations in MATBG. In particular, what is the origin of the soft magnetic excitations? Such soft collective modes have been predicted in insulting states of MATBG ${ }^{25-27}$. However, our experiments do not exhibit insulating behavior near $v=1$. One can imagine such soft modes emerging also in a gapless state, either as low-energy magnetic collective excitations of itinerant electrons, or due to a partial localization of some of the electrons, coexisting with other itinerant ones. The existence of such free moments at low temperatures should have far-reaching consequences for the transport behavior and the phase diagram of this fascinating strongly correlated electron system. 


\section{References}

1. Pomeranchuk, I. On the thery of He3. Zh.Eksp.Teor.Fiz 20, 919 (1950).

2. Bistritzer, R. \& MacDonald, A. H. Moiré bands in twisted double-layer graphene. Proc. Natl. Acad. Sci. 108, 12233-12237 (2011).

3. Cao, Y. et al. Correlated insulator behaviour at half-filling in magic-angle graphene superlattices. Nature 556, 80-84 (2018).

4. Cao, Y. et al. Unconventional superconductivity in magic-angle graphene superlattices. Nature 556, 43-50 (2018).

5. Li, G. et al. Observation of Van Hove singularities in twisted graphene layers. Nat. Phys. 6, 109-113 (2010).

6. Suárez Morell, E., Correa, J. D., Vargas, P., Pacheco, M. \& Barticevic, Z. Flat bands in slightly twisted bilayer graphene: Tight-binding calculations. Phys. Rev. B 82, 121407 (2010).

7. Regan, E. C. et al. Mott and generalized Wigner crystal states in WSe2/WS2 moiré superlattices. Nature 579, 359-363 (2020).

8. Tang, Y. et al. Simulation of Hubbard model physics in WSe2/WS2 moiré superlattices. Nature 579, 353-358 (2020).

9. Yankowitz, M. et al. Tuning superconductivity in twisted bilayer graphene. Science 363, 1059-1064 (2019).

10. Lu, X. et al. Superconductors, orbital magnets and correlated states in magic-angle bilayer graphene. Nature 574, 653-657 (2019).

11. Nuckolls, K. P. et al. Strongly Correlated Chern Insulators in Magic-Angle Twisted Bilayer Graphene. arXiv 2007.03810 (2020).

12. Wu, S., Zhang, Z., Watanabe, K., Taniguchi, T. \& Andrei, E. Y. Chern Insulators and Topological Flat-bands in Magic-angle Twisted Bilayer Graphene. ArXiv 2007.03725 (2020).

13. Das, I. et al. Symmetry broken Chern insulators and magic series of Rashba-like 
Landau level crossings in magic angle bilayer graphene. Arxiv 2007.13390 (2020).

14. Sharpe, A. L. et al. Emergent ferromagnetism near three-quarters filling in twisted bilayer graphene. Science 365, 605-608 (2019).

15. Serlin, M. et al. Intrinsic quantized anomalous Hall effect in a moiré heterostructure. Science 367, 900-903 (2020).

16. Kerelsky, A. et al. Maximized electron interactions at the magic angle in twisted bilayer graphene. Nature 572, 95-100 (2019).

17. Xie, Y. et al. Spectroscopic signatures of many-body correlations in magic-angle twisted bilayer graphene. Nature 572, 101-105 (2019).

18. Jiang, Y. et al. Charge order and broken rotational symmetry in magic-angle twisted bilayer graphene. Nature 573, 91-95 (2019).

19. Choi, Y. et al. Electronic correlations in twisted bilayer graphene near the magic angle. Nat. Phys. 15, 1174-1180 (2019).

20. Tomarken, S. L. et al. Electronic Compressibility of Magic-Angle Graphene Superlattices. Phys. Rev. Lett. 123, 046601 (2019).

21. Zondiner, U. et al. Cascade of phase transitions and Dirac revivals in magic-angle graphene. Nature 582, 203-208 (2020).

22. Po, H. C., Zou, L., Vishwanath, A. \& Senthil, T. Origin of Mott insulating behavior and superconductivity in twisted bilayer graphene. Phys. Rev. X 8, 031089 (2018).

23. Song, Z. et al. All Magic Angles in Twisted Bilayer Graphene are Topological. Phys. Rev. Lett. 123, 036401 (2019).

24. Ahn, J., Park, S. \& Yang, B.-J. Failure of Nielsen-Ninomiya Theorem and Fragile Topology in Two-Dimensional Systems with Space-Time Inversion Symmetry: Application to Twisted Bilayer Graphene at Magic Angle. Phys. Rev. X 9, 021013 (2019).

25. Bultinck, N. et al. Ground State and Hidden Symmetry of Magic-Angle Graphene at Even Integer Filling. Phys. Rev. X 10, 031034 (2020).

26. MacDonald, A. H. private communication. 
27. Wu, F. \& Das Sarma, S. Collective Excitations of Quantum Anomalous Hall Ferromagnets in Twisted Bilayer Graphene. Phys. Rev. Lett. 124, 046403 (2020).

28. Wong, D. et al. Cascade of electronic transitions in magic-angle twisted bilayer graphene. Nature 582, 198-202 (2020).

29. Spivak, B. \& Kivelson, S. A. Phases intermediate between a two-dimensional electron liquid and Wigner crystal. Phys. Rev. B 70, 155114 (2004).

30. Continentino, M. A., Ferreira, A. S., Pagliuso, P. G., Rettori, C. \& Sarrao, J. L. Solid state Pomeranchuk effect. Phys. B Condens. Matter 359-361, 744-746 (2005).

31. Pustogow, A. et al. Quantum spin liquids unveil the genuine Mott state. Nat. Mater. 17, 773-777 (2018).

32. Kuntsevich, A. Y., Tupikov, Y. V, Pudalov, V. M. \& Burmistrov, I. S. Strongly correlated two-dimensional plasma explored from entropy measurements. Nat. Commun. 6, 7298 (2015).

33. Hartman, N. et al. Direct entropy measurement in a mesoscopic quantum system. Nat. Phys. 14, 1083-1086 (2018).

34. Park, J. M., Cao, Y., Watanabe, K., Taniguchi, T. \& Jarillo-Herrero, P. Flavour Hund's Coupling, Correlated Chern Gaps, and Diffusivity in Moir'e Flat Bands. Arxiv 2008.12296 (2020).

35. Chen, S. et al. Electrically tunable correlated and topological states in twisted monolayer-bilayer graphene. Arxiv 2004.11340 (2020).

36. Spivak, B. \& Kivelson, S. A. Transport in two dimensional electronic microemulsions. Ann. Phys. (N. Y). 321, 2071-2115 (2006).

37. Saito, Y. et al. Isospin Pomeranchuk effect and the entropy of collective excitations in twisted bilayer graphene. ArXiv 2008.10830 (2020).

38. Uri, A. et al. Mapping the twist-angle disorder and Landau levels in magic-angle graphene. Nature 581, 47-52 (2020). 
Acknowledgements: We thank Ehud Altman, Eva Andrei, Eslam Khalaf, Steve Kivelson, Sankar Das Sarma, Gal Shavit, Joey Sulpizio, Senthil Todadri, Aviram Uri, Ashvin Vishwanath, Michael Zaletel and Eli Zeldov for useful suggestions. We note that a very recent work ${ }^{37}$ proposed, based on transport measurements, that a similar effect to that discussed here occurs near $v=-1$. In this work, in contrast, we measured the entropy directly, and mapped the entire phase diagram near $v=1$ using compressibility measurements. Work at Weizmann was supported by the Leona M. and Harry B. Helmsley Charitable Trust grant, ISF grants (712539 \& 13335/16), Deloro award, Sagol WeizmannMIT Bridge program, the ERC-Cog (See-1D-Qmatter, no. 647413), the ISF Research Grants in Quantum Technologies and Science Program (994/19 \& 2074/19), the DFG (CRC/Transregio 183), ERC-Cog (HQMAT, no. 817799), EU Horizon 2020 (LEGOTOP 788715) and the Binational Science Foundation (NSF/BMR-BSF grant 2018643). Work at MIT was primarily supported by the US Department of Energy (DOE), Office of Basic Energy Sciences (BES), Division of Materials Sciences and Engineering under Award DESC0001819 (J.M.P.). Help with transport measurements and data analysis were supported by the National Science Foundation (DMR-1809802), and the STC Center for Integrated Quantum Materials (NSF Grant No. DMR-1231319) (Y.C.). P.J-H acknowledges support from the Gordon and Betty Moore Foundation's EPiQS Initiative through Grant GBMF9643. The development of new nanofabrication and characterization techniques enabling this work has been supported by the US DOE Office of Science, BES, under award DE-SC0019300. K.W. and T.T. acknowledge support from the Elemental Strategy Initiative conducted by the MEXT, Japan, Grant Number JPMXP0112101001, JSPS KAKENHI Grant Numbers JP2OH00354 and the CREST(JPMJCR15F3), JST. This work made use of the Materials Research Science and Engineering Center Shared Experimental Facilities supported by the National Science Foundation (DMR-0819762) and of Harvard's Center for Nanoscale Systems, supported by the NSF (ECS-0335765). 
Contributions: A.R., J.M.P, U.Z., Y.C., P.J-H. and S.I. designed the experiment. A.R.,U.Z., performed the scanning SET experiments, J.M.P, Y.C. performed the monolayer graphene sensing experiments. D.R-L. and Y.C. fabricated the twisted bilayer graphene devices. A.R., J.M.P, U.Z., Y.C., P.J-H. and S. I. analyzed the data. E.B., Y.O. and A.S. wrote the theoretical model. K.W. and T.T. supplied the hBN crystals. A.R., J.M.P, U.Z., Y.C., Y.O., A.S., E.B., P.JH. and S.I. wrote the manuscript. 
a

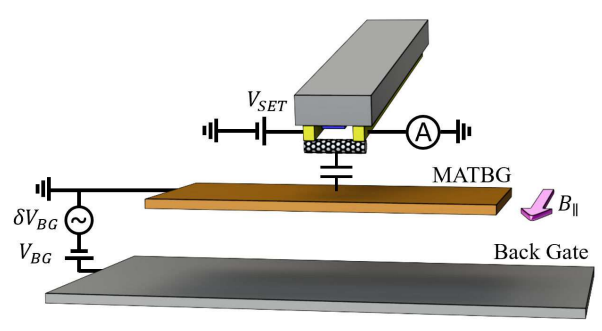

b

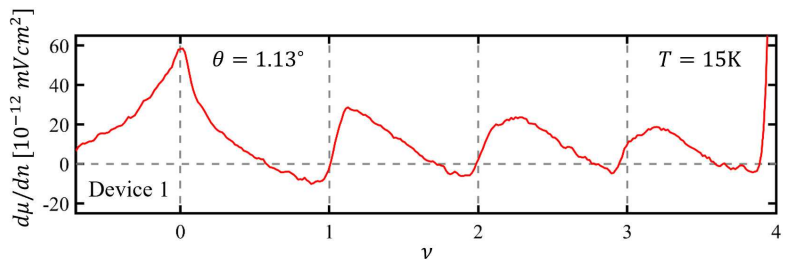

C

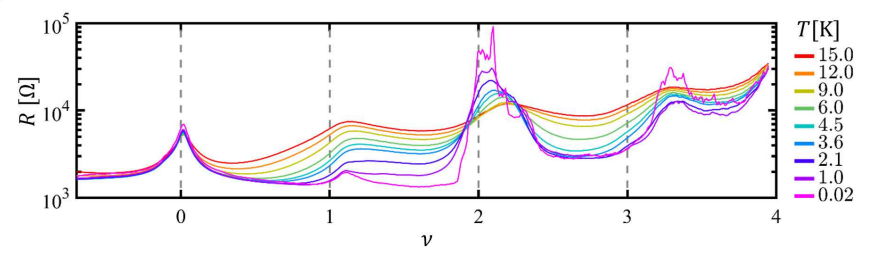

Figure 1: Experimental setup and device characterization. a. A nanotube-based single electron transistor (SET) is used to measure the local electronic compressibility and entropy of magic angle twisted bilayer graphene (MATBG). The MATBG is encapsulated between top and bottom h-BN layers (not shown) and has a metallic back-gate. By monitoring the current through the SET, we track changes in the MATBG chemical potential, $d \mu$, in response to a density modulation, $d n$, produced by an a.c. voltage on the back-gate ${ }^{21}$, $\delta V_{B G}$. A d.c. back-gate voltage, $V_{B G}$, sets the overall carrier density in the MATBG, $n$. Some of the measurements are performed in a parallel magnetic field, $B_{\|}$(indicated). b. Inverse compressibility, $d \mu / d n$, measured as a function of the moiré lattice filling factor, $v=n /\left(n_{s} / 4\right)$, at $T=15 K\left(n_{s}\right.$ is the density that correspond to 4 electrons per moiré site). Measurements are done on a large spatial domain $(\sim 5 \mu \mathrm{m} \times 4 \mu \mathrm{m})$ throughout which the twist angle is extremely homogenous, $\theta=1.130^{\circ} \pm 0.005$ (measured by spatial mapping of the $V_{B G}$ that corresponds to $n_{s}$, as in Refs. ${ }^{21,38}$ ). As seen previously ${ }^{21}$, a jump of $d \mu / d n$ appears near all integer filling factors. This jump corresponds to a Fermi surface reconstruction, in which some combination of the spin/valley flavors filling is reset back to near the charge neutrality point, and correspondingly $d \mu / d n$ shows a cascade of sawtooth features as a function of density. The trace is measured at $T=15 \mathrm{~K}$, showing that even at this high temperature this sawtooth cascade is well developed $\mathbf{c}$. Two-probe resistance, $R$, measured as a function of $v$ and temperature. Notice that unlike the inverse compressibility, which measures a local quantity, the resistance gives an averaged result over domains with different twist angle. Therefore, the resistance maxima are slightly shifted from the usual integer $v$ values, probably because another domain with a small difference in twist angle dominates the transport characteristics globally. 
a

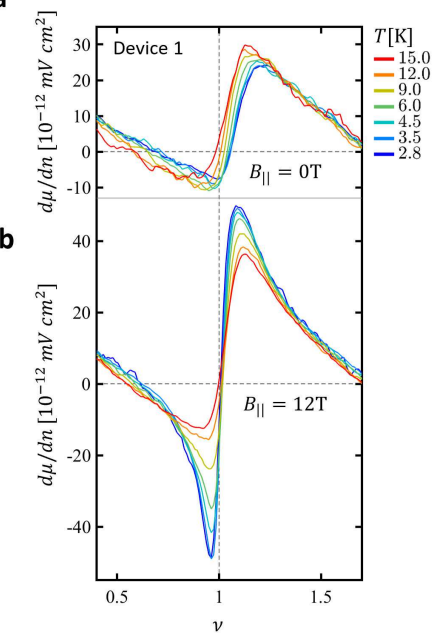

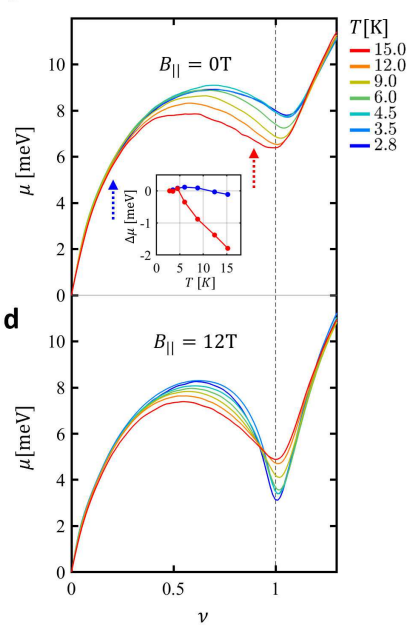

e

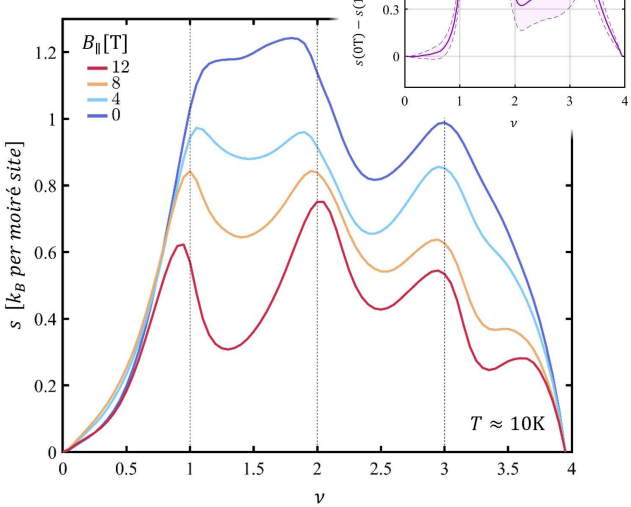

Figure 2: Measurement of large magnetic entropy above $\boldsymbol{v}=1$. a. Inverse compressibility, $d \mu / d n$, as a function of $v$, near $v=1$, measured at zero parallel magnetic field, $B_{\|}=0 T$, and at several temperatures. With increasing $T$, the jump in $d \mu / d n$ moves toward lower $v$ and becomes stronger. b. Same measurement done at $B_{\|} \approx 12 T$. Here, opposite to the zero-field case, increasing $T$ reduces the magnitude of the $d \mu / d n$ jump, as expected from thermal smearing. c. The chemical potential $\mu(v)$ (relative to that of the charge neutrality point) at $B_{\|}=0 T$, obtained by integrating the $d \mu / d n$ signal in panel a with respect to $n$. Inset: $\mu(T, v)-\mu(T=2.8 K, v)$ for $v=0.2$ (blue) and $v=0.9$ (red). At $v=0.2$ the chemical potential is nearly temperature independent, whereas at $v=0.9$ it is roughly constant until $T \sim 4 \mathrm{~K}$ and then start decreasing approximately linearly with $T$. d. Similar to c, but at $B_{\|}=12 T$. In contrast to the zero-field case, here, below $v \approx 0.9, \mu$ decreases with $T$ while above $v \approx 0.9 \mu$ increases with $T$. e. The electronic entropy in units of $k_{B}$ per moiré unit cell, as a function of $v$ at $T \approx 10 \mathrm{~K}$ and at various parallel magnetic fields, $B_{\|}=0,4,8,12 \mathrm{~T}$. To obtain the entropy we determine the partial derivative $(\partial \mu / \partial T)_{\nu, B_{\|}}$from a linear fit to the measured $\mu$ vs. $T$ in the range $T=4.5 \mathrm{~K}-15 \mathrm{~K}$. The entropy per moiré cell is then obtained by integrating Maxwell's relation: $(\partial s / \partial v)_{T, B_{\|}}=-(\partial \mu / \partial T)_{\nu, B_{\|}}$, over $v$ (see Supp Info. for details). At $B_{\|}=0$ the entropy climbs rapidly near $v=1$ to a value of $1.2 k_{B}$ per moiré cell. Inset: the difference between the entropies at low and high fields, $s\left(B_{\|}=0 \mathrm{~T}\right)-s\left(B_{\|}=12 \mathrm{~T}\right)$. The purple shading shows the estimated error bar. 
a

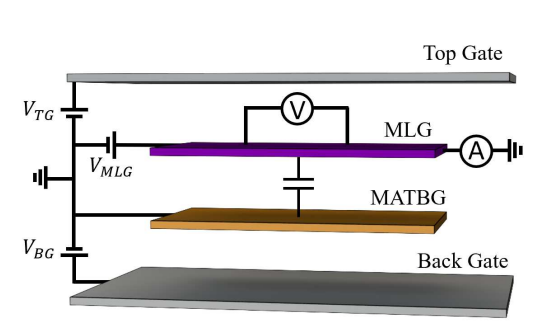

b

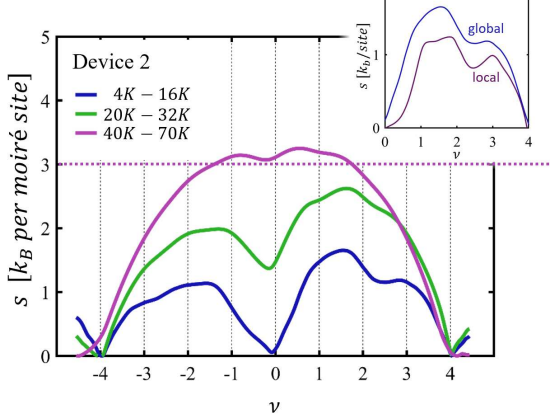

C

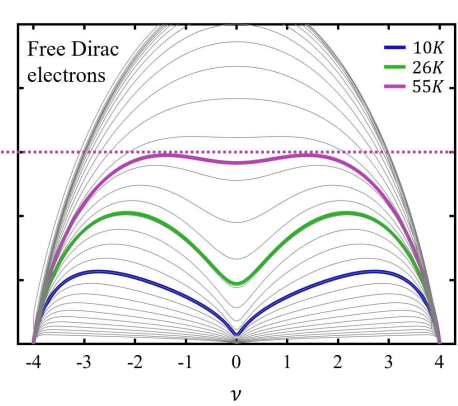

Figure 3: Temperature dependence of the Entropy. a. Experimental setup for measuring the global entropy, averaged over the entire device ${ }^{34}$. The device consists of MATBG and a monolayer graphene (MLG) sensor layer, separated by an ultrathin $(1 \mathrm{~nm})$ layer of $\mathrm{h}-\mathrm{BN}$ (not shown), as well as top and bottom metallic gates. By balancing the electrochemical potential of the adjacent layers in the device, we can obtain the relationship between the density and chemical potential of MATBG and MLG and the gate voltages applied to the system. In the special case where the density of MLG is zero, i.e. at its charge neutrality point, the chemical potential of MATBG is directly proportional to the voltage applied to the top gate. This technique allows us to reliably extract the chemical potential and entropy of MATBG at temperatures up to $70 \mathrm{~K}$. b. The measured entropy, in units of $k_{B}$ per moiré unit cell, as a function of $v$ at three different temperature ranges (top legend). The entropy derivative, $d s / d v$, is obtained from a linear fit to $\mu$ vs. $T$ in the corresponding temperature range, and is then integrated over $v$ to yield the entropy per moiré unit cell (similar to Fig. 2e). Inset: comparison between the $v$ dependences of the entropies, measured at the low temperature range, obtained from local and global measurements. c. The entropy as a function of $v$ and $T$ calculated for a system of four degenerate non-interacting Dirac bands (whose density of states climbs linearly with energy from the Dirac point to the end of the conduction or the valence band). The colorcoded lines show the curves whose temperatures correspond to the mean of the temperature ranges of the experimental curves. The gray lines represent the entire evolution from zero temperature to high temperature, where the entropy saturates on a value of $8 \ln (2) \approx 5.5$, where the factor 8 reflects the total number of energy bands. A bandwidth of $W=30 \mathrm{meV}$ is chosen such that the calculated value of the entropy at the highest temperature roughly matches the one obtained from the measured curve at the same temperature. 


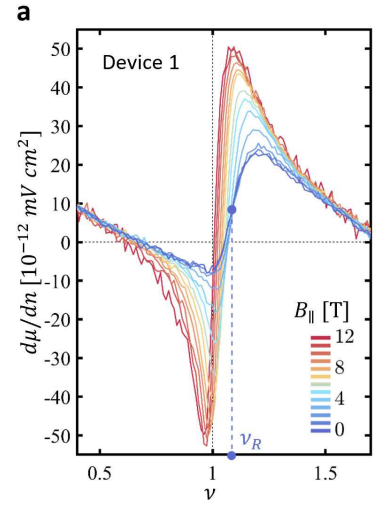

c

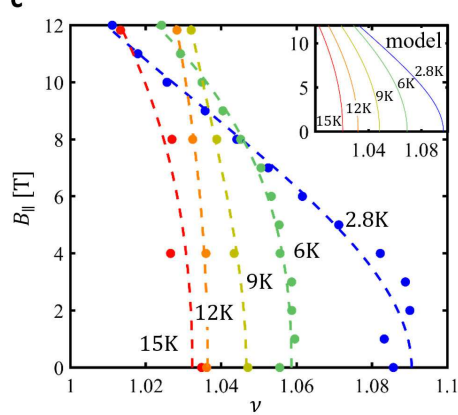

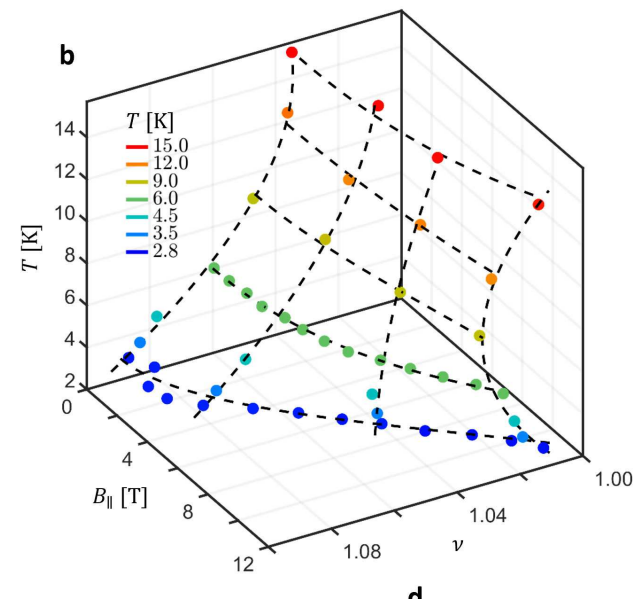

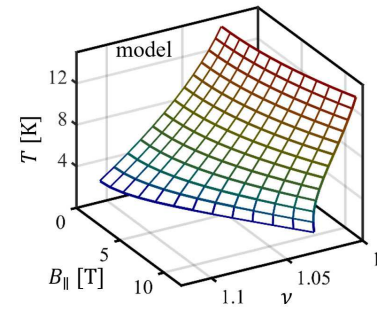

d

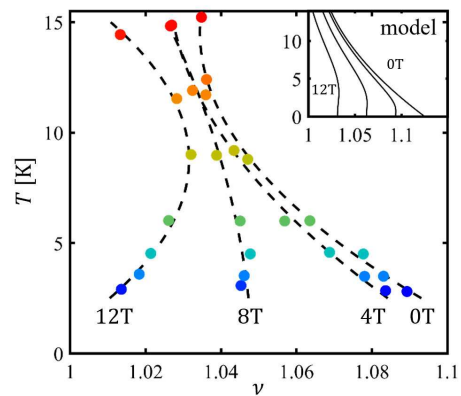

Figure 4: Experimental phase diagram. a. The inverse compressibility, $d \mu / d n$, measured as a function of $v$ near $v=1$, at several values of parallel magnetic field, $B_{\|}$. We track the filling factor that corresponds to the center the jump in $d \mu / d n$ (labeled $v_{R}$ ). Visibly, the application of $B_{\|}$pushes $v_{R}$ to lower values. b. Measured $v_{R}$ as a function of $B_{\|}$and $T$, plotted as dots in the $\left(v, B_{\|}, T\right)$ space (the dots are colored by their temperature). The dashed lines are polynomial fits to the dots at constant $B_{\|}$or constant $T$. Inset: the same surface calculated from a simple model that assumes a transition between a Fermi liquid and a metallic phase that contains one free moments per moiré site (see text). c. Projection of the data in panel b onto the $\left(v, B_{\|}\right)$plane, showing the dependence of $v_{R}$ on $B_{\|}$for various temperatures. At low fields, $v_{R}$ is independent of field but it becomes linear in $B_{\|}$at high fields, a behavior expected from the field polarization of free moments (see text). Inset: curves calculated from the model. d. Projection onto the $(v, T)$ plane, showing the dependence of $v_{R}$ on $T$ for various magnetic fields. At $B_{\|}=0 T, v_{R}$ is linear in $T$ at small $T$ 's and then curves up at higher $T$ 's. At high magnetic field, the dependence of $v_{R}$ on $T$ becomes non-monotonic. Inset: curves calculated from the model. 


\section{Figures}

a

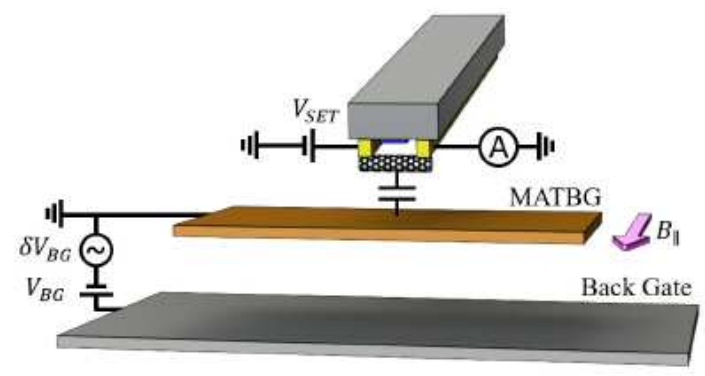

b

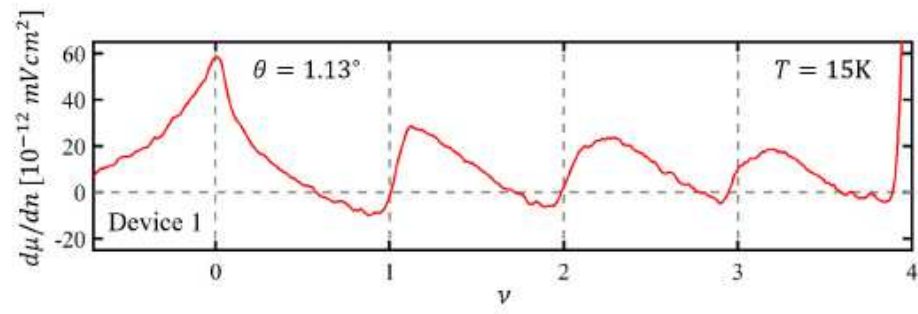

c

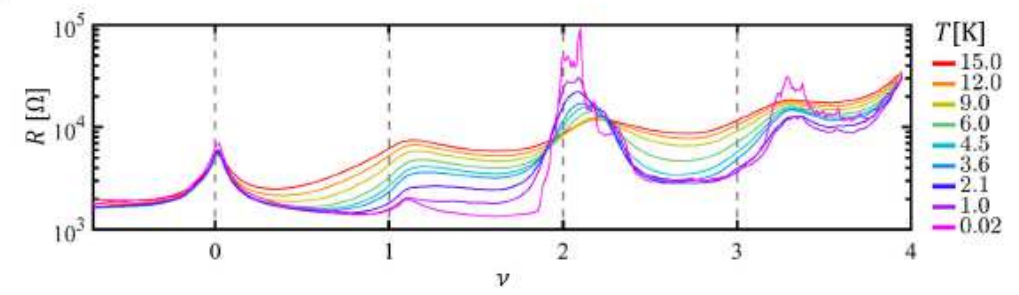

Figure 1

Experimental setup and device characterization. a. A nanotube-based single electron transistor (SET) is used to measure the local electronic compressibility and entropy of magic angle twisted bilayer graphene (MATBG). The MATBG is encapsulated between top and bottom h-BN layers (not shown) and has a metallic back-gate. By monitoring the current through the SET, we track changes in the MATBG chemical potential, $\mathrm{d} \mu$, in response to a density modulation, $\mathrm{dn}$, produced by an a.c. voltage on the back-gate21, ðVBG. A d.c. back-gate voltage, VBG, sets the overall carrier density in the MATBG, $n$. Some of the measurements are performed in a parallel magnetic field, B囚 (indicated). b. Inverse compressibility, $d \mu / d n$, measured as a function of the moiré lattice filling factor, $U=n /(n c / 4)$, at $T=15 \mathrm{~K}$ ( $\mathrm{nc}$ is the density that correspond to 4 electrons per moiré site). Measurements are done on a large spatial domain $(\sim 5 \mu \mathrm{N} \times$ $4 \mu \mathrm{N}$ ) throughout which the twist angle is extremely homogenous, $8=1.130 \otimes \pm 0.005$ (measured by spatial mapping of the VBG that corresponds to nc, as in Refs. 21,38). As seen previously21, a jump of $\mathrm{d} \mu / \mathrm{dn}$ appears near all integer filling factors. This jump corresponds to a Fermi surface reconstruction, in which some combination of the spin/valley flavors filling is reset back to near the charge neutrality point, and correspondingly $d \mu / d n$ shows a cascade of sawtooth features as a function of density. The trace is measured at $T=15 \mathrm{~K}$, showing that even at this high temperature this sawtooth cascade is well developed c. Two-probe resistance, $R$, measured as a function of $v$ and temperature. Notice that unlike the inverse compressibility, which measures a local quantity, the resistance gives an averaged result over domains with different twist angle. Therefore, the resistance maxima are slightly shifted from the usual integer $U$ values, probably because another domain with a small difference in twist angle dominates the transport characteristics globally. 
a

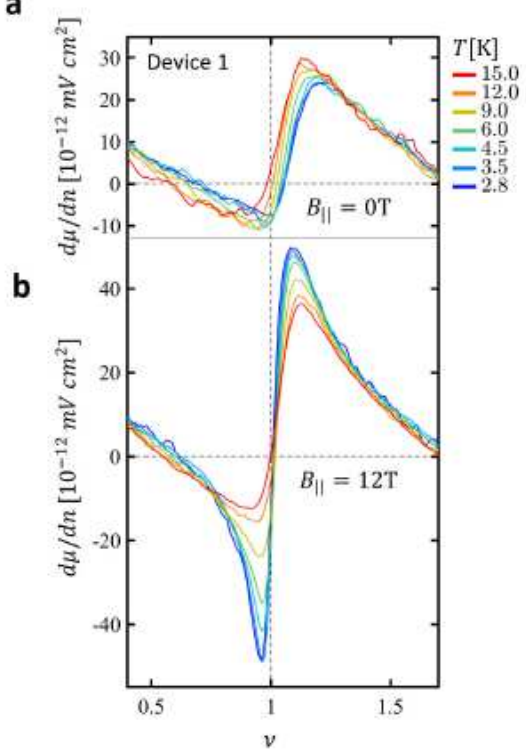

C

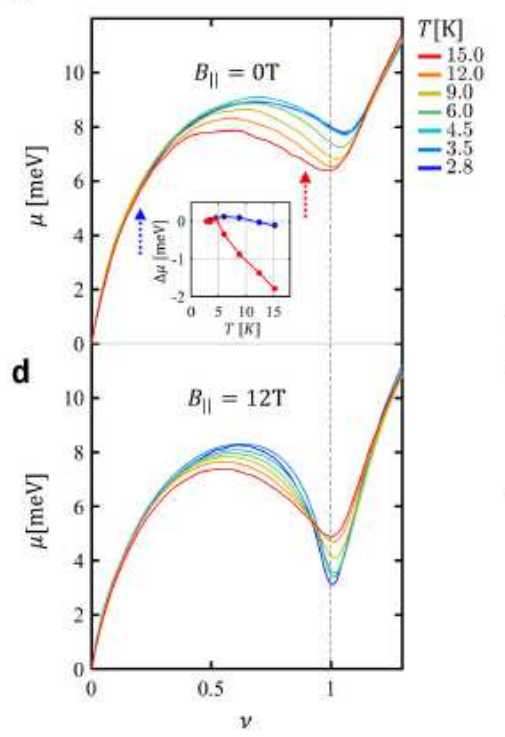

e

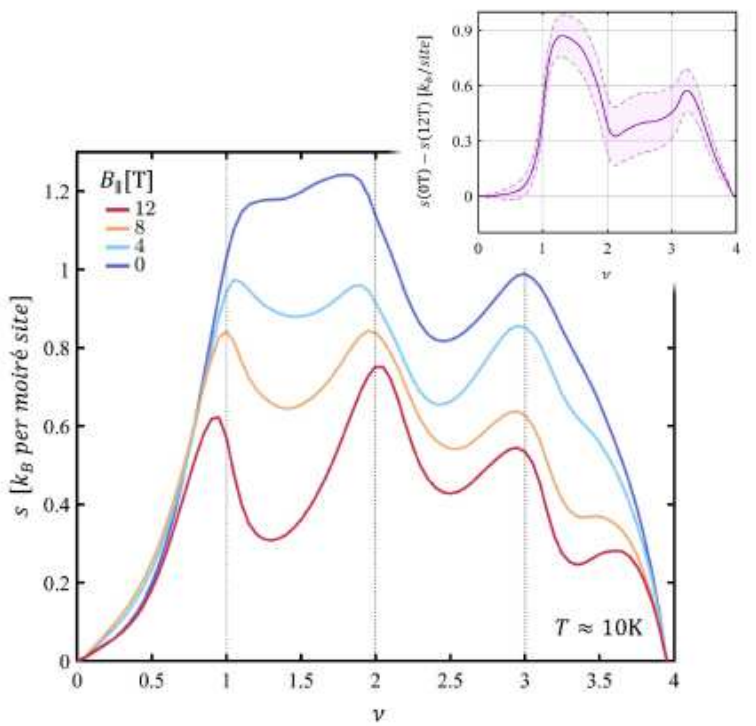

Figure 2

Measurement of large magnetic entropy above $U=1$. a. Inverse compressibility, $d \mu / d n$, as a function of $U$, near $U=1$, measured at zero parallel magnetic field, $B \otimes=0 T$, and at several temperatures. With increasing $T$, the jump in $d \mu / d n$ moves toward lower $U$ and becomes stronger. $b$. Same measurement done at $B \nabla \approx$ 12T. Here, opposite to the zero-field case, increasing $T$ reduces the magnitude of the $\mathrm{d} \mu / \mathrm{dn}$ jump, as expected from thermal smearing. c. The chemical potential $\mu(v)$ (relative to that of the charge neutrality point) at $\mathrm{B} \square=0 \mathrm{~T}$, obtained by integrating the $d \mu / d n$ signal in panel a with respect to $\mathrm{n}$. Inset: $\mu(\mathrm{T}, \mathrm{v})-\mu(\mathrm{T}$ $=2.8 \mathrm{~K}, \mathrm{v}$ ) for $\mathrm{v}=0.2$ (blue) and $\mathrm{v}=0.9$ (red). At $\mathrm{v}=0.2$ the chemical potential is nearly temperature independent, whereas at $\mathrm{v}=0.9$ it is roughly constant until $\mathrm{T} \otimes 4 \mathrm{~K}$ and then start decreasing approximately linearly with $\mathrm{T}$. $\mathrm{d}$. Similar to $\mathrm{c}$, but at $\mathrm{B} \nabla=12 \mathrm{~T}$. In contrast to the zero-field case, here, below $v \approx 0.9, \mu$ decreases with $T$ while above $v \approx 0.9 \mu$ increases with $T$. e. The electronic entropy in units of $k B$ per moiré unit cell, as a function of $v$ at $T \approx 10 \mathrm{~K}$ and at various parallel magnetic fields, $\mathrm{B} \triangle=0,4,8,12 \mathrm{~T}$. To obtain the entropy we determine the partial derivative $(\partial \mu / \partial T) \mathrm{v}, \mathrm{B} \otimes$ from a linear fit to the measured $\mu$ vs. $\mathrm{T}$ in the range $\mathrm{T}=4.5 \mathrm{~K}-15 \mathrm{~K}$. The entropy per moiré cell is then obtained by integrating Maxwell's

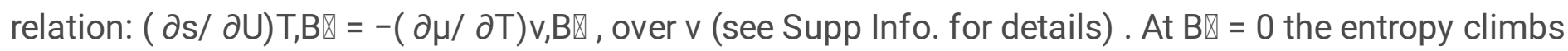
rapidly near $\mathrm{v}=1$ to a value of $1.2 \mathrm{kB}$ per moiré cell. Inset: the difference between the entropies at low and high fields, $s(B \otimes=0 T)-s(B \otimes=12 T)$. The purple shading shows the estimated error bar. 
a

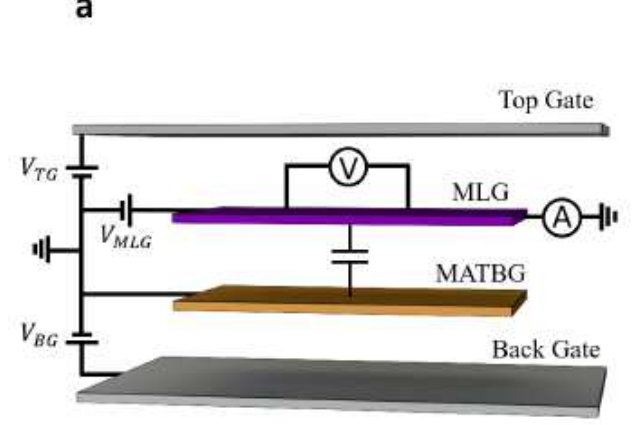

b

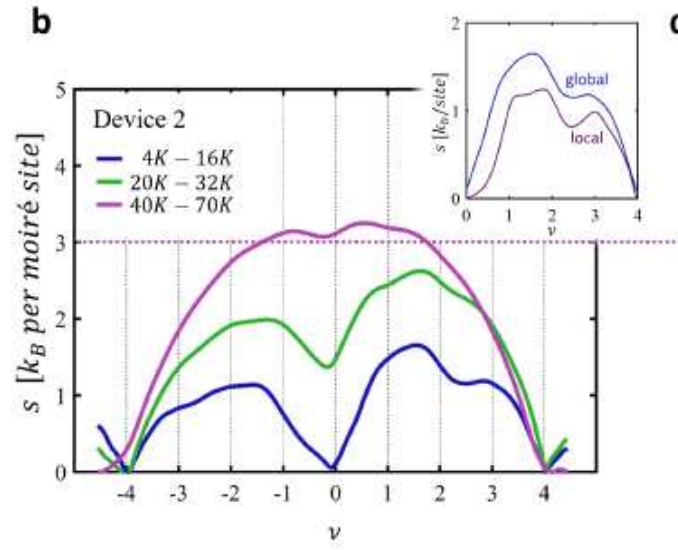

c

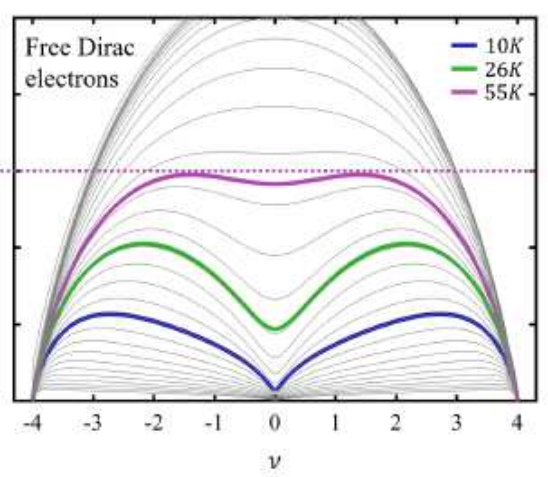

\section{Figure 3}

Temperature dependence of the Entropy. a. Experimental setup for measuring the global entropy, averaged over the entire device34. The device consists of MATBG and a monolayer graphene (MLG) sensor layer, separated by an ultrathin ( $1 \mathrm{nN}$ ) layer of h-BN (not shown), as well as top and bottom metallic gates. By balancing the electrochemical potential of the adjacent layers in the device, we can obtain the relationship between the density and chemical potential of MATBG and MLG and the gate voltages applied to the system. In the special case where the density of MLG is zero, i.e. at its charge neutrality point, the chemical potential of MATBG is directly proportional to the voltage applied to the top gate. This technique allows us to reliably extract the chemical potential and entropy of MATBG at temperatures up to $70 \mathrm{~K}$. b. The measured entropy, in units of kB per moiré unit cell, as a function of $U$ at three different temperature ranges (top legend). The entropy derivative, $d s / d v$, is obtained from a linear fit to $\mu$ vs. $T$ in the corresponding temperature range, and is then integrated over $v$ to yield the entropy per moiré unit cell (similar to Fig. 2e). Inset: comparison between the v dependences of the entropies, measured at the low temperature range, obtained from local and global measurements. c. The entropy as a function of $v$ and $T$ calculated for a system of four degenerate non-interacting Dirac bands (whose density of states climbs linearly with energy from the Dirac point to the end of the conduction or the valence band). The color- coded lines show the curves whose temperatures correspond to the mean of the temperature ranges of the experimental curves. The gray lines represent the entire evolution from zero temperature to high temperature, where the entropy saturates on a value of $8 \ln (2) \approx 5.5$, where the factor 8 reflects the total number of energy bands. A bandwidth of $W=30 \mathrm{meV}$ is chosen such that the calculated value of the entropy at the highest temperature roughly matches the one obtained from the measured curve at the same temperature. 


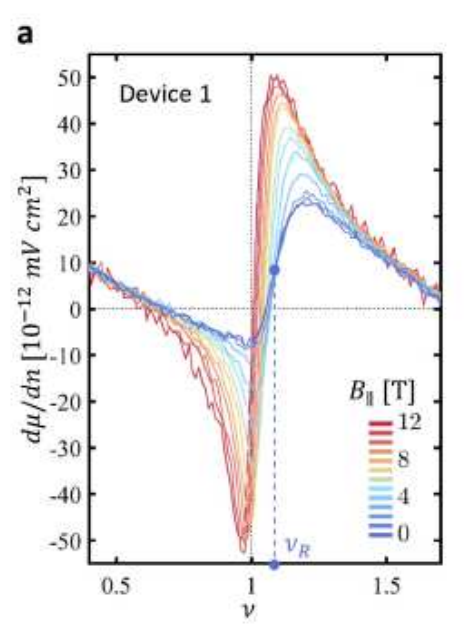

c

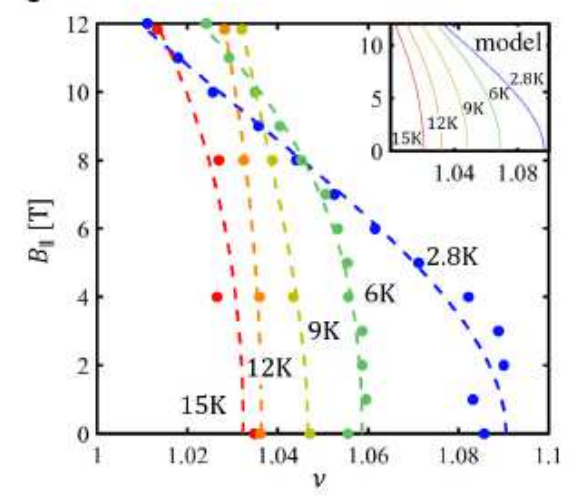

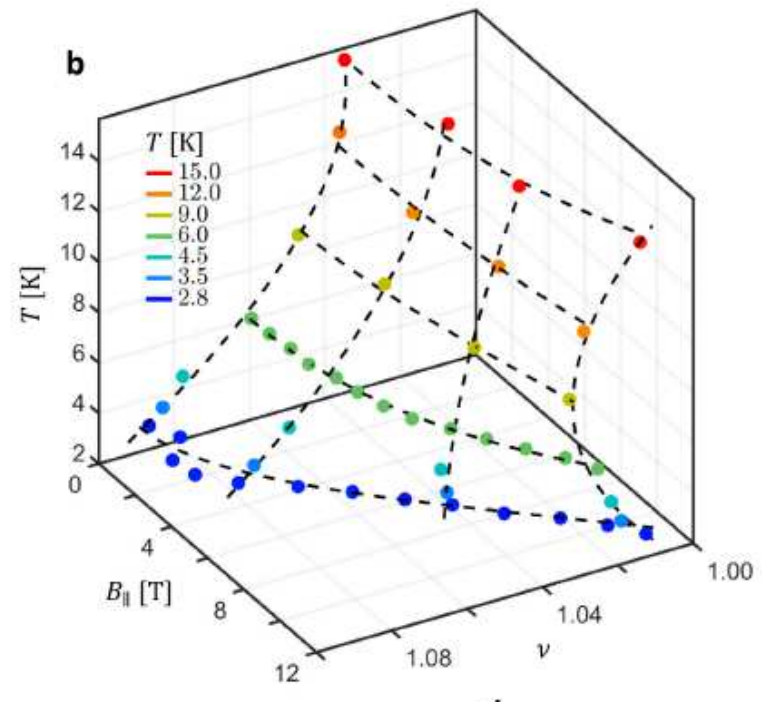

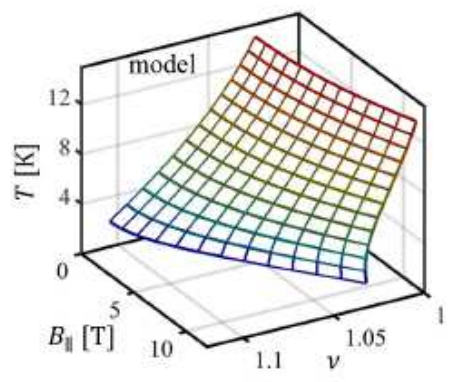

d

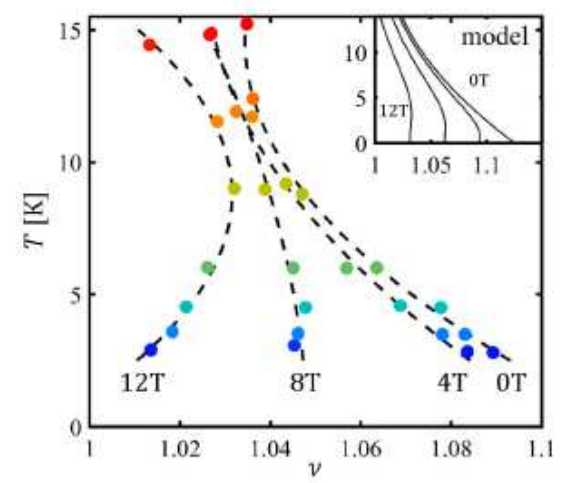

\section{Figure 4}

Experimental phase diagram. a. The inverse compressibility, $d \mu / d n$, measured as a function of $U$ near $U=$ 1 , at several values of parallel magnetic field, B囚. We track the filling factor that corresponds to the center the jump in $d \mu / d n$ (labeled UR ). Visibly, the application of B囚 pushes UR to lower values. b. Measured UR as a function of $B \bigotimes$ and $T$, plotted as dots in the $(U, B \otimes, T)$ space (the dots are colored by their temperature). The dashed lines are polynomial fits to the dots at constant $\mathrm{B} \bigotimes$ or constant $\mathrm{T}$. Inset: the same surface calculated from a simple model that assumes a transition between a Fermi liquid and a metallic phase that contains one free moments per moiré site (see text). c. Projection of the data in panel $b$ onto the $(\mathrm{U}, \mathrm{B} \bigotimes)$ plane, showing the dependence of UR on $\mathrm{B} \otimes$ for various temperatures. At low fields, UR is independent of field but it becomes linear in $\mathrm{B} \bigotimes$ at high fields, a behavior expected from the field polarization of free moments (see text). Inset: curves calculated from the model. d. Projection onto the (v, $T$ ) plane, showing the dependence of UR on $T$ for various magnetic fields. At $B \otimes=0 T$, UR is linear in $T$ at small $T$ 's and then curves up at higher T's. At high magnetic field, the dependence of UR on T becomes non-monotonic. Inset: curves calculated from the model.

\section{Supplementary Files}


This is a list of supplementary files associated with this preprint. Click to download.

- SuppInfoHighQualityPDF.pdf 